\title{
Generalized (k, r) - Lucas Numbers
}

\author{
Ashwini Panwar \\ School of Studies in \\ Mathematics, Vikram \\ University Ujjain, India
}

\author{
Kiran Sisodiya \\ School of Studies in \\ Mathematics, Vikram \\ University Ujjain, India
}

\author{
G.P.S. Rathore \\ Department of Mathematics \\ College of Horticulture, \\ Mandsaur, India
}

\begin{abstract}
In this paper, we have defined new kinds of $(k, r)$-Lucas number. But the difference among these sequences comes to the forefront not only through the value of the natural number $\mathrm{k}$ but also through the value of new parameter which we find involved in the definition of this distance. Consequently we have various properties of these numbers for study.
\end{abstract}

\section{General Terms}

Number theory, Fibonacci numbers, Lucas numbers, Pell numbers, Golden Ratio, Golden Sections, Applied Mathematics et. al..

\section{Keywords}

$\mathrm{k}$ - Lucas Number, (k, r) Lucas Number, Binet`s Formula, Generating engines.

\section{INTRODUCTION}

Classic Fibonacci numbers and classic Lucas numbers have charismatic properties and researcher have been shown their generalization and implemented in every field of science and arts. Classical Lucas numbers have been generalized in various way [6]. One of these generalizations that have been of a great interest lately among mathematical researchers is one that paves a way to the $\mathrm{k}$ - Lucas number.

\subsection{Definition}

For every natural numbers, the k- Lucas sequence $L_{k}=\left\{L_{k, n}\right\}$ is defined by the recurrence relation[6]

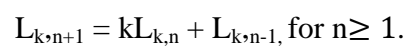

with initial conditions $\mathrm{L}_{\mathrm{k}, 0}=2, \mathrm{~L}_{\mathrm{k}, 1}=\mathrm{k}$

From above definition the general expression of first k- Lucas numbers are presented in Table 1:

Table 1: k - Lucas numbers

\begin{tabular}{|c|}
\hline $\mathrm{L}_{\mathrm{k}, 1}=\mathrm{k}$ \\
\hline $\mathrm{L}_{\mathrm{k}, 2}=\mathrm{k}^{2}+2$ \\
\hline $\mathrm{L}_{\mathrm{k}, 3}=\mathrm{k}^{3}+3 \mathrm{k}$ \\
\hline $\mathrm{L}_{\mathrm{k}, 4}=\mathrm{k}^{4}+4 \mathrm{k}$ \\
\hline $\mathrm{L}_{\mathrm{k}, 5}=\mathrm{k}^{5}+5 \mathrm{k}^{3}+5 \mathrm{k}$ \\
\hline $\mathrm{L}_{\mathrm{k}, 6}=\mathrm{k}^{6}+6 \mathrm{k}^{4}+9 \mathrm{k}^{2}+$ \\
2 \\
\hline $\mathrm{L}_{\mathrm{k}, 7}=\mathrm{k}^{7}+7 \mathrm{k}^{5}+14 \mathrm{k}^{3}$ \\
$+7 \mathrm{k}$ \\
\hline
\end{tabular}

If $\mathrm{k}=1$, the classical Lucas sequence is obtained $\{2,1,3,4,7$, $11,18, .$.

If $\mathrm{k}=2$, the Pell - Lucas sequence is obtained $\{2,2,6,14,34$, $82,198, \ldots\}$

\section{GENERALIZED $(k, r)$ LUCAS NUMBERS}

This section witnesses the application of the definition of $r$ distance to the $\mathrm{k}$ - Lucas numbers in a such peculiar way that it generalizes the earlier findings $[2,4]$. The intensely appealing formula are those which are used to calculate the general term of the sequences produced by the above definition. Moreover it supports in finding out the sum of the first $\mathrm{n}$ terms.

\subsection{Definition}

We define the generalized $(\mathrm{k}, \mathrm{r})$ Lucas number $L_{k, n}(r)$ for the natural numbers $\mathrm{k} \geq 1, n \geq 0, r \geq 1$ by the recurrence relation

$$
L_{k, n}(r)=k L_{k, n-r}(r)+L_{k, n-2}(r) \text { for } n \geq r
$$

with the initial conditions

$$
L_{k, n}(r)=2, n=0,1,2, \ldots, r-1 \text {, except } L_{k, 1}(1)=k .
$$

So, $\quad$ if $L_{k}(r)=\left\{L_{k, n}(r) / n \in N\right\}$, the expressions of the sequences obtained for $r=1,2, \ldots, 5$.

$$
\begin{gathered}
L_{k}(1)=\left\{2, k, k^{2}+2, k^{3}+3 k, k^{4}+4 k^{2}+2, k^{5}+5 k^{3}+\right. \\
\left.5 k, k^{6}+6 k^{4}+9 k^{2}+2, k^{7}+7 k^{5}+14 k^{3}+7 \mathrm{k}, \ldots\right\} \\
L_{k}(2)=\left\{2,2,2 k+2,2 k+2,2 k^{2}+4 k+2,2 k^{2}+4 k\right. \\
\left.+2,2 k^{3}+6 k^{2}+6 k+2, \ldots\right\} \\
L_{k}(3)=\left\{2,2,2,2 k+2,2 k+2,4 k+2,2 k^{2}+4 k+2,2 k^{2}\right. \\
+6 k+2, \ldots\} \\
L_{k}(4)=\left\{2,2,2,2,2 k+2,2 k+2,4 k+2,4 k+2,2 k^{2}+6 k\right. \\
+ \\
\left.2,2 k^{2}+6 k+2, \ldots\right\} \\
L_{k}(5)=\{2,2,2,2,2,2 k+2,2 k+2,4 k+2,4 k+2,6 k \\
+
\end{gathered}
$$

$2.2(k, r)-$ Lucas Numbers for $k=1,2,3$ For $\mathrm{k}=1,2,3$, we obtain distinct integers sequences which is For $\mathrm{k}=1$, the following sequences are obtained

Table $2(1, r)$-Lucas numbers

\begin{tabular}{c|cccccccc}
$\mathrm{n}$ & 0 & 1 & 2 & 3 & 4 & 5 & 6 & $7 \ldots$ \\
\hline$L_{1, n}(1)$ & 2 & 1 & 3 & 4 & 7 & 11 & 18 & 29 \\
$L_{1, n}(2)$ & 2 & 2 & 4 & 4 & 8 & 8 & 16 & $16 \ldots$ \\
$L_{1, n}(3)$ & 2 & 2 & 2 & 4 & 4 & 6 & 8 & $10 \ldots$ \\
$L_{1, n}(4)$ & 2 & 2 & 2 & 2 & 4 & 4 & 6 & $6 \ldots$ \\
$L_{1, n}(5)$ & 2 & 2 & 2 & 2 & 2 & 4 & 4 & $6 \ldots$
\end{tabular}


$\mathrm{L}_{1}(1)$ is the classical Lucas sequence $\mathrm{L}=\left\{\mathrm{L}_{\mathrm{n}}\right\}=\{2,1,3,4$, $7,11,18,29, \ldots\}$

Table 3 (2, r) Lucas Numbers

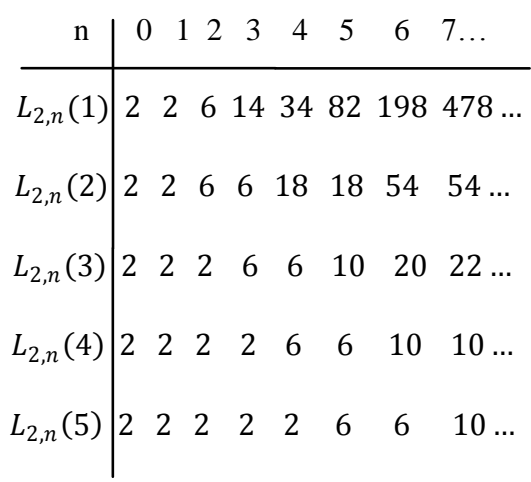

Table 4 (3, r) Lucas Number

\begin{tabular}{c|cccccccc}
$\mathrm{n}$ & 0 & 1 & 2 & 3 & 4 & 5 & 6 & $7 \ldots$ \\
\hline$L_{3, n}(1)$ & 2 & 3 & 11 & 36 & 119 & 393 & $1298 \ldots$ \\
$L_{3, n}(2)$ & 2 & 2 & 8 & 8 & 32 & 32 & 128 & $128 \ldots$ \\
$L_{3, n}(3)$ & 2 & 2 & 2 & 8 & 8 & 14 & 32 & $38 \ldots$ \\
$L_{3, n}(4)$ & 2 & 2 & 2 & 2 & 8 & 8 & 14 & $14 \ldots$ \\
$L_{3, n}(5)$ & 2 & 2 & 2 & 2 & 2 & 8 & 8 & $14 \ldots$
\end{tabular}

\section{PROPERTIES OF $(k, r)$}

\section{GENERALIZED LUCAS SEQUENCE}

\subsection{First Explicit Formula for (k, r) -}

Generalized Lucas Numbers

The French mathematician Binet's dropped out two analytical formulas for the Fibonacci and Lucas numbers. In our case, Binet's formula favors us to express the generalized $(k, r)$ Lucas numbers in function of the roots $\alpha_{1}$ and $\alpha_{2}$ of the following characteristic equation, associated to the recurrence relation

$$
\begin{aligned}
& \alpha^{2}-k \alpha-1=0(3) \\
& \text { where } \alpha_{1}=\frac{k+\sqrt{k^{2}+4}}{2}, \alpha_{2}=\frac{k-\sqrt{k^{2}+4}}{2}, \\
& \alpha_{1}+\alpha_{2}=k, \\
& \alpha_{1}-\alpha_{2}=\sqrt{k^{2}+4}, \alpha_{1} \alpha_{2}=-1 .
\end{aligned}
$$

\section{Proposition 1.(Binet's Formula)}

The $n^{\text {th }}$ Generalized (k, r) Lucas number is given by

$$
L_{k, n}(r)=\alpha_{1}^{n}+\alpha_{2}^{n} \text {. }
$$

Proof.

Since equation (3) has two distinct roots, then the sequence is

$$
L_{k, n}(r)=C_{1} \alpha_{1}^{n}+C_{2} \alpha_{2}^{n}(5)
$$

is the solution of the equation (3), Giving to $n$ the values $n=$ 0,1 and $\mathrm{r}=1,2$ respectively. Then we get values $C_{1}=$ $C_{2}=1$

using in (5),we get

$$
L_{k, n}(r)=\alpha_{1}^{n}+\alpha_{2}^{n} \text {. }
$$

\subsection{Catalan`s Identity}

In 1879 a Belgian Mathematician Eugene Charles Catalan inaugurated the Catalan`s identity. Now we introduce Catalan`s identity for $(k, r)$ Lucas number.

Proposition 2. (Catalan`s Identity)

$L_{k, n-m}(r) . L_{k, n+m}(r)-L_{k, n}^{2}(r)=(-1)^{n-m} L_{k, 2 m}(r)+$
$2(-1)^{n+1}$.

Proof.

Using Binet`s formula, we get

$$
\begin{aligned}
& L_{k, n-m}(r) . L_{k, n+m}(r)-L_{k, n}^{2}(r)=\left[\alpha_{1}^{n-m}+\alpha_{2}^{n+m}\right] \cdot\left[\alpha_{1}^{n+m}+\right. \\
& \left.\alpha_{2}^{n+m}\right]-\left[\alpha_{1}^{n}+\alpha_{2}^{n}\right]^{2} \\
& =(-1)^{n-m}\left[\alpha_{1}^{2 m}+\alpha_{2}^{2 m}\right]+2(-1)^{n+1} \\
& =(-1)^{n-m} L_{k, 2 m}(r)+\mathbf{2}(-1)^{n+1} \text {. }
\end{aligned}
$$

\subsection{Cassini`s Identity}

Jean-Dominique Cassini brought to light this identity in 1680 .

Proposition 3. (Cassini`s Identity )

$L_{k, n-1}(r) \cdot L_{k, n+1}(r)-L_{k, n}^{2}(r)=(-1)^{n-1} L_{k, 2}(r)+$
$2(-1)^{n+1}$

Proof.

In (6), put $\mathrm{m}=1$, to get above result.

\subsection{D`Ocagne`s Identity}

Proposition 4. (d`Ocagne`s Identity )

If $m>n$, then

$L_{k, m}(r) . L_{k, n+1}(r)-L_{k, m+1}(r) . L_{k, n}(r)=$

$(-1)^{n}\left(\sqrt{k^{2}+4}\right)\left[L_{k, m}(r)-\left(k+\sqrt{k^{2}+4}\right)^{m-n}\right.$.

Proof.

$L_{k, m}(r) \cdot L_{k, n+1}(r)-L_{k, m+1}(r) \cdot L_{k, n}(r)=\left[\alpha_{1}^{m}+\right.$ $\left.\alpha_{2}^{m}\right] \cdot\left[\alpha_{1}^{n+1}+\alpha_{2}^{n+1}\right]-\left[\alpha_{1}^{m+1}+\alpha_{2}^{m+1}\right] \cdot\left[\alpha_{1}^{n}+\alpha_{2}^{n}\right]$

$=\left(\alpha_{1} \alpha_{2}\right)^{n} \cdot\left(\alpha_{1}-\alpha_{2}\right)\left(\alpha_{2}^{m-n}-\alpha_{1}^{m-n}\right)$

$=(-\mathbf{1})^{n}\left(\sqrt{k^{2}+4}\right)\left[L_{k, m}(r)-2\left(\alpha_{1}\right)^{m-n}\right.$

$=(-1)^{n}\left(\sqrt{k^{2}+4}\right)\left[L_{k, m}(r)-\left(k+\sqrt{k^{2}+4}\right)^{m-n}\right.$.

\subsection{Limit of the two consecutive terms}

In this section we break down that the limit of the quotient of two consecutive terms is equal to the positive root of the corresponding characteristic equation.

Proposition 5.

$\lim _{n \rightarrow \infty} \frac{L_{k, n+1}(r)}{L_{k, n}(r)}=\alpha_{1}$.

Proof. We have

$$
\begin{aligned}
\lim _{n \rightarrow \infty} \frac{L_{k, n+1}(r)}{L_{k, n}(r)} & =\lim _{n \rightarrow \infty}\left(\frac{\alpha_{1}^{n+1}+\alpha_{2}^{n+1}}{\alpha_{1}^{n}+\alpha_{2}^{n}}\right) \\
& =\lim _{n \rightarrow \infty} \frac{\alpha_{1}^{n+1}}{\alpha_{1}^{n}}\left(\frac{1+\left(\frac{\alpha_{2}}{\alpha_{1}}\right)^{n+1}}{1+\left(\frac{\alpha_{2}}{\alpha_{1}}\right)^{n}}\right)
\end{aligned}
$$

Since $\alpha_{2}<\alpha_{1}$, then $\lim _{n \rightarrow \infty}\left(\frac{\alpha_{2}}{\alpha_{1}}\right)^{n}=0$,

We get

$\lim _{n \rightarrow \infty} \frac{L_{k, n+1}(r)}{L_{k, n}(r)}=\alpha_{1}$. 
Also it can be shown easily

\section{Proposition 6.}

$\lim _{n \rightarrow \infty} \frac{L_{k, n}(r)}{L_{k, n+1}(r)}=\frac{1}{\alpha_{1}}$.

Proof .

It can be also shown like Proposition 5 .

\subsection{Generating function for the $(k, r)$ - Generalized Lucas Number}

In below paragraph, the generating function for the $(\mathrm{k}, \mathrm{r})$ Lucas sequences is given.

\section{Proposition 7.}

Generating function of sequence

of $L_{k}(r)=\left\{L_{k . n}(r)\right\}$ is given by

$\mathbf{G}\left(L_{k . n}(r): x\right)=\frac{2+2 x}{1-x^{2}-k x^{r}}$.

Proof.

$$
\begin{aligned}
& \mathrm{G}\left(L_{k . n}(r): x\right)= \sum_{n=0}^{\infty} L_{k . n}(r) x^{n} \\
&= L_{k .0}(r) x^{0}+L_{k .1}(r) x^{1}+L_{k .2}(r) x^{2}+ \\
& L_{k .3}(r) x^{3}+\cdots \\
&= 2+2 \mathrm{x}+\sum_{n=2}^{\infty} L_{k . n}(r) x^{n} \\
&= 2+2 \mathrm{x}+\sum_{n=2}^{\infty}\left[k L_{k . n-r}(r)+L_{k . n-2}(r)\right] x^{n} \\
&= 2+2 \mathrm{x}+\mathrm{k} x^{r} \sum_{n=2}^{\infty} k L_{k . n-r}(r) x^{n-r}+ \\
& x^{2} \sum_{n=2}^{\infty} L_{k . n-2}(r) x^{n-2} \\
&=2+2 \mathrm{x}+\mathrm{k} x^{r} \sum_{j=0}^{\infty} k L_{k . p}(r) x^{p}+x^{2} \sum_{j=o}^{\infty} L_{k . j}(r) x^{j}
\end{aligned}
$$

where $\mathrm{p}=\mathrm{n}-\mathrm{r}, \mathrm{n}=0,1,2,3, \ldots$ and $\mathrm{r}=1,2,3, \ldots$ and $\mathrm{j}=\mathrm{n}-$

so $\left(1-\mathrm{k} x^{r}-x^{2}\right) \sum_{n=0}^{\infty} L_{k . n}(r) x^{n}=2+2 \mathrm{x}$ we have $L_{k . n}(r)=\frac{2+2 x}{1-x^{2}-k x^{r}}$.

\section{ACKNOWLEDGEMENT}

We would like to thank the anonymous referees for numerous helpful suggestions

\section{REFERENCES}

[1] B. Singh, P. Bhadouria and O. Sikhwal, Sum Properties for the k- Lucas number with arithmetic indexes, , J. Math. Comput. Sci., 4(2014), 105- 117.

[2] D. Brod, K. Piejko and I. Wloch, Distance Fibonacci numbers, distance Lucas numbers and their applications, ArsCombinatoria, CXII(2013), 397- 410 .

[3] D. Tasci and E. Kilic, On the order k- generalized Lucas Numbers, Appl. Math. Comput. 155(2014), 637- 64.

[4] I. Wloch, U. Bednarz, D. Brod, A. Wloch and M Wolowiecz-Musial, On a new type of distance Fibonacci numbers, Discrete Applied Mathematics,161(2013), 2695-2701

[5] K. Keygisiz and A. Sahin, New generalizations of Lucas numbers, Gen. Math. Notes, , 10(1) (2012), 63-77.

[6] S. Falcon and A. Plaza, On the k- Lucas numbers, international Journal of Contemporary Mathematical Sciences, 21(6) (2011), 1039-1050.

[7] S. Falcon, On k-Lucas numbers of arithmetic indexes,Applied Mathematics and Computation, 3(2012), 1202- 1206.

[8] S. Falcon, On Lucas triangle and its relationship with kLucas numbers, J. Math. Comput. Sci. 2(2012), 425-34.

[9] Y.K.Gupta, V.H.Badshah, M.Singh, K.Sisodiya Diagonal Function of k-Lucas Polynomial, Turkish Journal of Analysis and Number Theory 3(2)(2015), 4952 